\title{
TAGUNG
}

\section{Unbekannt, ungewollt, unnötig? Die EU-Reflexionsgruppe „Horizont 2020-2030“6}

\author{
Frédéric Krumbein*
}

Die Arbeit der EU-Reflexionsgruppe „Horizont 2020-2030“ zur Zukunft der Europäischen Union stand im Mittelpunkt der Tagung, die am 26. und 27. November $2009 \mathrm{im}$ Stuttgarter Rathaus vom „Europa Zentrum Baden-Württemberg“ und dem „Arbeitskreis Europäische Integration" unter wissenschaftlicher Leitung von Gabriele Abels und Michèle Knodt veranstaltet wurde.

Die EU-Reflexionsgruppe unter der Präsidentschaft des früheren spanischen Ministerpräsidenten Felipe González Márquez ist beauftragt, dem Europäischen Rat bis Juni 2010 einen Bericht mit einer Vision zur Zukunft der Europäischen Union vorzulegen. Dazu wurde ein zwölfköpfiger ,Rat der Weisen“ einberufen, dem der Oberbürgermeister von Stuttgart, Wolfgang Schuster, als einziges Mitglied aus Deutschland angehört. Die Gruppe beschäftigt sich mit einzelnen Politikfeldern und den Herausforderungen, die auf Staat und Gesellschaft in diesen Feldern in der Europäischen Union in den Jahren 2020 bis 2030 zukommen. Vor diesem Hintergrund waren die Schwerpunkte in den einzelnen Vorträgen der Tagung gesetzt worden.

Am Anfang der Tagung stand der Bericht von Wolfgang Schuster und Rainer Münz, beide Mitglieder der aktuellen Reflexionsgruppe. In einem ersten Teil befasste sich die Tagung sodann mit Reflexionsgruppen als Instrument der Europapolitik in historischer Perspektive und ihren Erfolgsbedingungen. Im zweiten Teil der Tagung ging es um die Zukunft des ,multi-level governance'-Systems und des

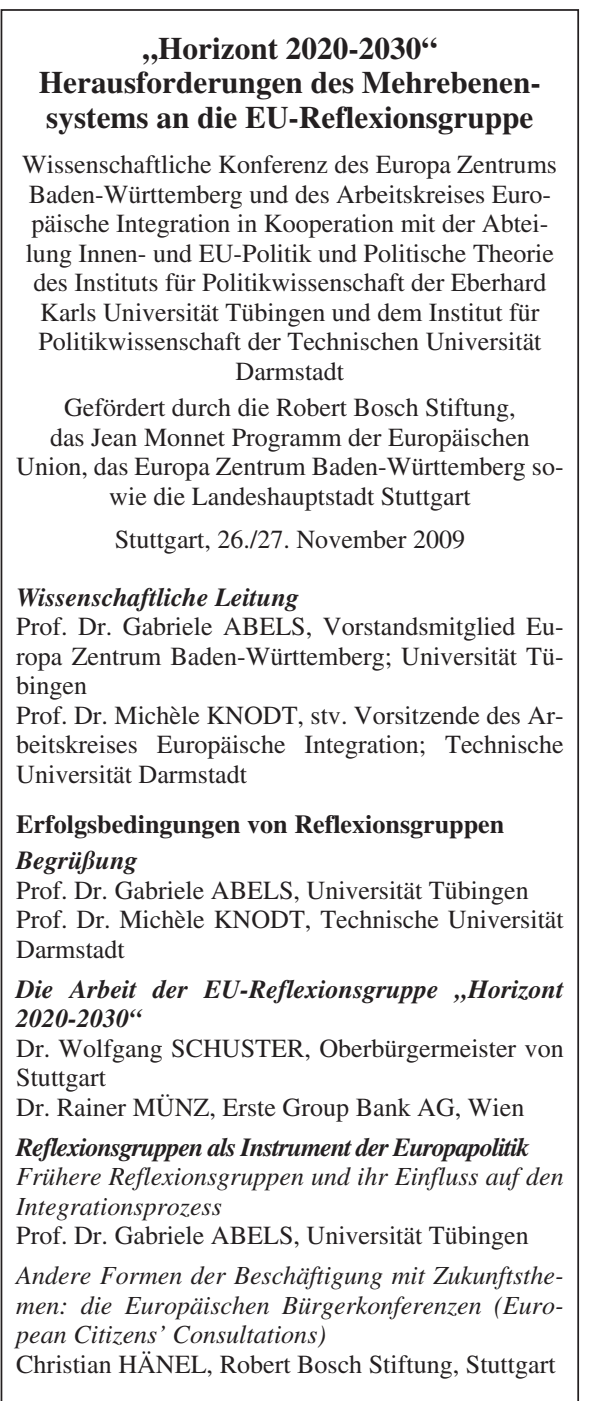

* Frédéric Krumbein, Geschäftsführer des Arbeitskreises Europäische Integration e.V., Berlin. 
Subsidiaritätsprinzips und um zukünftige Herausforderungen des kommunalen Regierens in Europa. Der dritte Teil der Tagung widmete sich schließlich einzelnen Politikfeldern, an denen die zuvor erarbeiteten Thesen anschaulich gemacht wurden.

Die Arbeit der EU-Reflexionsgruppe „Horizont 2020-2030“

Wolfgang Schuster, Mitglied der Reflexionsgruppe „Horizont 2020-2030“, führte in Entstehung, Arbeit und Ziele der aktuellen Reflexionsgruppe ein. Die Hauptaufgaben der Reflexionsgruppe seien das Nachdenken über die langfristigen Perspektiven Europas (für die Jahre 2020 bis 2030) und über die künftigen Herausforderungen. Die Reflexionsgruppe treffe sich einmal im Monat für eineinhalb bis zwei Tage. $\mathrm{Zu}$ den Treffen würden für einen Gedankenaustausch Experten und europäische Persönlichkeiten, wie Jacques Delors oder Valéry Giscard d'Estaing, eingeladen. Einige der Themen seien: erstens, Bürgernähe der Europäischen Union; zweitens, die Herausforderungen durch demografischen Wandel und Migration; drittens, die Modernisierung des europäischen Wirtschafts- und Sozialmodells; viertens, Energiesicherheit und Klimaschutz; fünftens, eine wirkungsvolle europäische Außen- und Sicherheitspolitik und Europas Rolle in der Welt sowie sechstens, die Legitimität der Europäischen Union. Das Ziel der Überlegungen und der Debatten der Gruppe sei ein stärkeres Europa. Dieses Ziel müsse bei gleichzeitiger Erweiterung der Europäischen Union und schwindender Popularität des europäischen Projektes vorangetrieben werden.

Rainer Münz, Mitglied der Reflexionsgruppe aus Österreich, führte am Beispiel Bevölkerungsentwicklung und Arbeitsmarkt in die Arbeit der Reflexionsgruppe ein. Er gab einen Überblick über demografische, migrationspolitische und beschäftigungspolitische Herausforderungen, die auf die Europäische Union zukommen werden. Die Europäische Union sei die zweitwichtigste Einwanderungsregion
Erfolgsbedingungen von Reflexionsgruppen, Verhandlungs- und Reformprozessen

Erfolgsbedingungen politischer Verhandlungsprozesse - Schlussfolgerungen für die Arbeit in der Reflexionsgruppe

Prof. Dr. Dr. h.c. Werner WEIDENFELD, LudwigMaximilians-Universität München

Erfolgsbedingungen lokaler und regionaler Interessenvermittlung in Reflexionsgruppen

Dr. Stefan NIEDERHAFNER, Visiting Professor, Ewha Womans University, Seoul

Herausforderungen im Mehrebenensystem

Politik im Mehrebenensystem vor dem Hintergrund der Reflexionsgruppe

Das EU-System des, Multi-Level Governance' angesichts zukünftiger Herausforderungen

Dr. des. Martin GROßE HÜTTMANN, Universität Tübingen

Wirksamkeit und Entwicklungsperspektiven des Subsidiaritätsprinzips in der EU

Prof. Dr. Rudolf HRBEK, Universität Tübingen

Herausforderungen des kommunalen Regierens im EU-Mehrebenensystem

Kommunales Regieren im europäischen Mehrebenensystem

Prof. Dr. Michèle KNODT, Technische Universität Darmstadt

Zukünftige Rolle des Ausschusses der Regionen als Vertreter der Kommunen und Regionen in der EU Dr. Gerhard STAHL, Generalsekretär des Ausschusses der Regionen, Brüssel

Kommunale Politik im Mehrebenensystem

Zukünftige demografische Entwicklungen im EUMehrebenensystem

Dr. Ole WINTERMANN, Bertelsmann Stiftung, Gütersloh

Klimapolitik im Mehrebenensystem

Annegret EPPLER, M.A., Ass. iur., Universität Tübingen

Neue Wege in der Sozial- und Beschäftigungspolitik im Mehrebenensystem - Herausforderungen für die kommunale Politik

Dr. Ute BEHNING, Institut für europäische Wohlfahrtssystemforschung, Bramsche

Die Rolle der kommunalen Ebene in der Identifikation der BürgerInnen mit der EU

Dr. Angelika VETTER, Universität Stuttgart

Diskussion: Schlussfolgerungen für die Arbeit in der Reflexionsgruppe

Prof. Dr. Gabriele ABELS, Universität Tübingen Prof. Dr. Michèle KNODT, Technische Universität Darmstadt

der Welt nach den USA. Diese Entwicklung sei ein neues Phänomen und in der Mentalität der Europäer noch nicht verankert. Über Jahr- 
hunderte hat Europa die Welt bevölkert, so Nordamerika, Australien und Neuseeland. Europa sei erst seit wenigen Jahrzehnten eine Einwanderungsregion. Inzwischen seien achtzig Prozent des Bevölkerungswachstums in der Europäischen Union migrationsbedingt. Eine steigende Lebenserwartung in der Europäischen Union führe zusammen mit niedrigen Kinderzahlen zu demografischen Problemen. In den nächsten Jahrzehnten werde es allein in Deutschland jährlich etwa eine halbe Million mehr Sterbefälle als Geburten geben. Nach Ansicht von Münz sollte die Europäische Union folgende Maßnahmen zur Bewältigung der demografischen Probleme ergreifen: Steigerung der Produktivität, Erhöhung des Rentenalters, Verringerung der Rente, Stabilisierung der Kinderzahl, Erweiterung der Europäischen Union und Förderung der Zuwanderung qualifizierter Migranten. Münz bemerkte, dass der von der Reflexionsgruppe festgestellte Handlungsdruck und die möglichen Lösungen politisch und gesellschaftlich möglicherweise nicht immer gewünscht seien. Abschließend ging Münz auf das der jüngeren Generation fehlende europäische Narrativ ein. Die Sicherung des Friedens und die Völkerverständigung würden inzwischen als selbstverständlich akzeptiert und könnten nicht mehr als Motivation für weitere Integrationsschritte dienen.

\section{Erfolgsbedingungen von Reflexionsgruppen}

Gabriele Abels legte ihrem Einführungsvortrag „Frühere Reflexionsgruppen und ihr Einfluss auf den Integrationsprozess" eine weite Definition von Reflexionsgruppen zugrunde: „Eine Gruppe, deren primärer Zweck darin bestehe, sich im kleinen, exklusiven Kreis, der Idee nach abgeschirmt von politischen Einflüssen und unabhängig vom alltäglichen ,policymaking', ausgestattet mit einem Mandat der EU-Institutionen über grundlegende integrationspolitische Projekte zu beratschlagen und zu reflektieren, um dem Integrations- prozess neue Impulse zu geben." Reflexionsgruppen hätten damit die primäre Aufgabe einer ,langfristigen politischen Agendaplanung" ". Abels beklagte, der Forschungsstand zum Thema Reflexionsgruppen sei bisher nicht zufriedenstellend. In einem nächsten Schritt definierte sie Reflexionsgruppen als spezifisches Instrument der Politikberatung. Anhand einer systematischen Analyse ausgewählter Reflexionsgruppen und ihrer $\mathrm{Ab}$ schlussberichte - vom „Spaak-Bericht“ (1956), über den „Davignon-Bericht“ (1970) und den „Tindemans-Bericht“ (1975) bis hin zu den Berichten von Delors (1989), Westendorp (1995) und Kok (2004) - stellte sie Gemeinsamkeiten dar und erarbeitete Kriterien, welche aus Perspektive der Forschung zur Politikberatung für den Erfolg von Reflexionsgruppen ausschlaggebend seien. Die Gemeinsamkeiten der bisher existierenden Reflexionsgruppen seien: Erstens, sie seien meistens vom Europäischen Rat eingesetzt worden; zweitens, die Vorsitzenden seien oft ehemalige Spitzenpolitiker aus einem der sechs Gründungsmitglieder der Gemeinschaft; drittens, die Reflexionsgruppen hätten meist ein breites Mandat; viertens, Bürgernähe sei in vielen Fällen ein Thema der Gruppen und fünftens, die Reflexionsgruppen hätten nach Ansicht von Abels langfristig eine relativ hohe Wirkung entfaltet. Die ausschlaggebenden Kriterien für den Erfolg von Reflexionsgruppen seien: erstens, Zeit und institutionelle Anbindung der Reflexionsgruppe; zweitens, Konsens in den Gruppen und in der Wissenschaft als Legitimierung der Ergebnisse; drittens, die Reputation der Ratgeber; viertens, die ,Verwertbarkeit" der Expertise, das heißt die Umsetzungsmöglichkeit in praktische Politik und fünftens, die Übereinstimmung der Ergebnisse mit den Interessen der Politik. Im Ergebnis stellte sie für die Arbeit der aktuellen Reflexionsgruppe keine erfolgversprechende Prognose: Das Timing der Gruppe sei, so kurz nach Inkrafttreten des Lissabon-Vertrags, eher ungünstig. Das Man-

1 Matthias Chardon/Dominik Hierlemann/Sarah Seeger: Den Weisen einen Rat, Bertelsmann Stiftung: spotlight europe 2007/07, S. 5. 
dat der Gruppe sei eingeschränkt und unscharf. Der politische Wille der Mitgliedstaaten hinsichtlich der Ergebnisse der Reflexionsgruppe sei unklar. Die heterogene Zusammensetzung der Gruppe sowie das Fehlen einer öffentlichen Debatte seien weitere Erfolgshindernisse.

Christian Hänel sprach über die Europäischen Bürgerkonferenzen als alternative Formen der Beschäftigung mit der Zukunft Europas. 1.600 zufällig ausgewählte Bürger diskutierten während zweitägiger Konferenzen im März 2009 in neun Mitgliedsländern über die Europäische Union. Der Prozess der Bürgerkonferenzen wurde durch Onlinedebatten ergänzt, an denen 250.000 Menschen teilnahmen. Im Mai 2009 gab es dann eine EUübergreifende Konferenz, die eine Bürgererklärung verabschiedete. In der Bürgererklärung seien fünfzehn Empfehlungen an die Europäische Union formuliert worden, unter anderem mehr europäische Aktivität in den Bereichen Bildung, Gesundheit, Klimaschutz und Finanzmarktregulierung. Der Mehrwert bei diesen Konferenzen lag vor allem in der zufälligen Auswahl der Teilnehmer. So führten sie Bürger aus vielen unterschiedlichen gesellschaftlichen Gruppen zusammen. Solche Bürgerkonferenzen seien ein mögliches Instrument, mit dessen Hilfe die Ergebnisse der Reflexionsgruppe in die Öffentlichkeit getragen werden könnten.

Die Konsequenzen aus der Arbeit von bisherigen Reflexionsgruppen für die aktuelle Gruppe waren das Thema des Vortrags von Werner Weidenfeld. Er analysierte das machtpolitische Umfeld und die Arbeit früherer Reflexionsgruppen sowie ihre Berichte, insbesondere den „Tindemans-“ und den „DelorsBericht“. Während Tindemans 1975/76 eine öffentliche Reflexion durchgeführt habe und zu einem Bericht gekommen sei, der zwar wissenschaftlich besprochen worden, politisch jedoch irrelevant gewesen sei, habe der strategische Bericht, den Delors zu Beginn seiner Amtszeit als Kommissionspräsident präsentiert habe, aus der zuvor herrschenden
,Eurosklerose“ geführt. Delors habe den Staats- und Regierungschefs zwei Projekte vorgeschlagen: entweder die Schaffung des europäischen Binnenmarktes oder die Realisierung einer effektiven europäischen Außenund Sicherheitspolitik. Der Europäische Rat sollte sich für eines der beiden Projekte entscheiden, weil der Europäischen Union die Kraft fehle beide Projekte voranzutreiben. Die Staats- und Regierungschefs entschieden sich für die Schaffung des Binnenmarktes. Jacques Delors verfügte mit François Mitterrand und Helmut Kohl über zwei wichtige Verbündete bei diesem bedeutenden Integrationsschritt. Insgesamt sei die Wirkung der bisherigen Reflexionsgruppen auf die europäische Integration eher gering gewesen. Viele der von den Gruppen vorgeschlagenen Reformen wären ohnehin durchgeführt worden - mit oder ohne Reflexionsgruppe.

Aus der Geschichte der europäischen Integration lassen sich folgende Erfolgsfaktoren für Reflexionsgruppen ableiten: Problemdruck müsse bestehen und auch wahrgenommen werden; strategisches und langfristiges Denken müsse die Arbeit bestimmen; politische Macht der Mitglieder müsse zur Umsetzung vorhanden sein; nicht zuletzt müsse eine politische Infrastruktur - Timing, Netzwerkfähigkeit, mediale Präsenz - bestehen. Erfüllt die Reflexionsgruppe „Horizont 2020-2030“ die genannten vier Kriterien? Die Probleme der Europäischen Union werden teilweise wahrgenommen und strategisches Denken sei vorhanden. Politische Macht und eine machtpolitische Infrastruktur fehlten aber bei der aktuellen Reflexionsgruppe. Die nicht vorhandene politische Macht der Reflexionsgruppe ergebe sich aus der ablehnenden Haltung der europäischen Staats- und Regierungschefs ihr gegenüber. Ihre Ergebnisse würden im besten Falle einen Impuls in Form von neuen Ideen erbringen, wie im Fall des „Tindemans-Berichtes“. Eine darüber hinausgehende Wirkung sei unwahrscheinlich. Weidenfeld merkte zum Schluss an, erfolgreiche Reflexionsgruppen hätten in der Geschichte der Integration ihr Mandat immer überschrit- 
ten, und riet der jetzigen Gruppe, dies auch zu tun. Die Reflexionsgruppe „Horizont 20202030" darf sich laut Mandat nur mit Inhalten der EU-Politik beschäftigen, nicht mit Institutionen und Prozessen der Union.

Stefan Niederhafners Vortrag über die Erfolgsbedingungen lokaler und regionaler Interessenvermittlung in Reflexionsgruppen betonte, für die lokale Ebene im europäischen Mehrebenensystem sei nicht nur der Aufbau eigener EU-Kompetenz, sondern auch eine möglichst frühzeitige Einbringung eigener Interessen in den EU-Entscheidungsprozess notwendig. Lokale Akteure hätten vor allem dann Möglichkeiten, sich in europäische Entscheidungen einzubringen, wenn sie den Institutionen ,Zugangsgüter ' präsentierten. Das könne gegenüber der Kommission etwa Wissen über bestehenden Handlungsbedarf, technische Umsetzungsmöglichkeiten oder die Bewertung einzelner Handlungsalternativen sein. Die lokale Ebene verfüge über solche Zugangsgüter aufgrund ihrer Erfahrung in der Umsetzung von EU-Politik und habe aufgrund ihrer Bürgernähe einen hohen Grad an Legitimation. Die abschließenden Empfehlungen von Niederhafner an die Städte und Regionen waren: Sie sollten deutlich europäischer planen und handeln, vermehrt Europakompetenz aufbauen und die Europäisierung ihrer Bürgerschaft vorantreiben. Städte und Regionen sollten frühzeitig im europäischen Mehrebenensystem EU-Politik betreiben und nicht erst, wenn der Entscheidungsprozess sich dem Ende zuneige.

\section{Zukünftige Herausforderungen im Mehrebe- nensystem}

Martin Große Hüttmann sprach über das EUSystem als System des, multi-level governance', des Regierens im Mehrebenensystem. Regieren im Mehrebenensystem meine, dass vier Ebenen zusammen regieren müssten: Europäische Union, Nationalstaat, regionale Ebene und Kommunen. Seiner Meinung nach hänge von der Organisation des Systems des ,multi-level governance‘ die Zukunftsfähig- keit der Europäischen Union ab. Er betonte, dass die politische Diskussion ,multi-level governance' ernst nehmen müsse und die unterschiedlichen politischen Ebenen nicht gegeneinander ausspielen dürfe. Sie müsse vielmehr eine Form von ,smart governance" entwickeln, die Effektivität und Effizienz des Gesamtsystems erhöhe. ,Multi-level governance' könne außerdem als Modell für ,meta governance' dienen, also die Frage, wie politische Steuerung in der Europäischen Union in eine bestimmte Richtung gelenkt werden könne. Für die Arbeit in der Reflexionsgruppe machte Große Hüttmann den Vorschlag, Sozialkapital zu europäisieren. Sozialkapital entstehe, wenn Gruppen sich bürgerschaftlich organisieren und dabei Vertrauen aufbauen, das wiederum die Demokratie und die Institutionen stärke. Gerade auf der lokalen Ebene gebe es Sozialkapital, wie Vereine, die stärker in die Europäische Union miteinbezogen werden sollten. Für die Politisierung der europäischen Politik müssen nach Große Hüttmann politische Räume geschaffen werden zur Austragung von politischen Streitfragen. Die Reflexionsgruppe, so seine Empfehlung, sollte offensiv und selbstbewusst das Modell des ,multi-level governance" nach innen und außen verteidigen.

Rudolf Hrbek referierte über die Wirksamkeit und die Perspektiven des Subsidiaritätsprinzips in der Europäischen Union. Im Subsidiaritätsprinzip würden zwei Prinzipien miteinander verbunden: Effizienz und politische Handlung auf der angemessenen Ebene. Die Europäische Union werde nach dem Subsidiaritätsprinzip nur tätig, wenn die Gemeinschaftsebene entweder handeln müsse (die nationale Ebene allein könne die Politik nicht gestalten und umsetzen) oder die Gemeinschaftsebene die Politik qualitativ besser umsetzen könne. Das Subsidiaritätsprinzip sei ein dynamisches Prinzip, weil es sowohl zur Erweiterung der Tätigkeit der EU-Kommission geeignet sei als auch zur Begrenzung der Tätigkeit der EU-Ebene. Durch den Vertrag von Lissabon werde das Subsidiaritätsprinzip nicht nur in seinem Bezug auf Regionen und 
Kommunen erweitert, sondern es werde mit dem sogenannten ,Frühwarnsystem ' und dem neuen Klagerecht auch ein Verfahren zur Subsidiaritätskontrolle eingerichtet. Kontrollberechtigt seien die nationalen Parlamente. Aus den neuen Regeln resultiert nach Hrbek eine Erhöhung der Legitimität des europäischen Gesetzgebungsprozesses, aber auch eine Zunahme der Komplexität. Abschließend gab Hrbek zu bedenken, dass das begrenzte Mandat der Reflexionsgruppe eine Weiterentwicklung des Subsidiaritätsprinzips nicht umfasse. Er kritisierte dieses Mandat als zu eng begrenzt, weil politische Inhalte nicht von politischen Institutionen und Prozessen getrennt werden könnten.

Michèle Knodt sprach über kommunales Regieren im europäischen Mehrebenensystem. Von regulativer Politik der Europäischen Union - etwa Daseinsvorsorge, Umwelt- oder Verkehrspolitik - und auch von distributiver EU-Politik, wie der Strukturfondsförderung, seien Kommunen betroffen. Eine Europäisierung der kommunalen Ebene finde durch eine ,Inkorporierung' von auf der EU-Ebene konsolidierten Normen, Werten und Regeln in die kommunale Politikgestaltung statt. Das europäische Mehrebenensystem sei ein polyzentrisches System mit unterschiedlichen, formal voneinander unabhängigen Entscheidungszentren und multiplen, überlappenden und lose gekoppelten Arenen. Entscheidungen beruhten in diesem polyzentrischen System auf Konsens, Interaktion und Kommunikation zwischen den Ebenen. Anhäufung von Wissen und Austausch von Ideen und Konzepten seien typische konsensorientierte Handlungsmuster. In diesem System agieren die Kommunen nach Knodt durch Anpassungsleistungen der lokalen Ebene an die EU-Politik, die direkte Vertretung ihrer Interessen auf der europäischen Ebene, die Institutionalisierung kommunaler Interessen im EU-/EG-Institutionengefüge, die nationale und regionale Vernetzung der kommunalen Akteure sowie der Konstruktion transnational-kommunaler Räume. Allerdings hänge die generelle Handlungsfähigkeit der kommunalen Akteure im
EU-Mehrebenensystem von ihrer verfassungsrechtlichen Stellung, ihren administrativen und finanziellen Ressourcen und ihrer strategischen Handlungsfähigkeit im EUMehrebenensystem ab. Während die kommunale Interessenrepräsentation sich bis Mitte der 1990er Jahre lediglich auf die Implementationsphase bezogen habe, umfasse sie nun alle Phasen des Policy-Zyklus vom AgendaSetting über die Politikentscheidung und Politikformulierung. Knodt gab der Reflexionsgruppe zu bedenken, dass die kommunale Ebene von allen Fragen, die in der Gruppe behandelt werden, betroffen sei. Sie regte an, zumindest prozedurale Fragen (wenn schon keine institutionellen) in der Reflexionsgruppe zu behandeln.

Gerhard Stahl, Generalsekretär des Ausschusses der Regionen (AdR), legte die besondere Rolle des AdR als Vertreter der Kommunen und Regionen im EU-Institutionengefüge dar. Er ging zunächst auf die heterogene Zusammensetzung des AdR mit Vertretern der lokalen und regionalen Ebene ein und betonte, im AdR seien Vertreter der exekutiven und der legislativen Gewalt vereint. Weiterhin erläuterte Stahl die Anhörungsrechte des AdR, die neuen Rechte nach dem Vertrag von Lissabon und die Politik seiner Stellungnahmen. Um mit anderen Akteuren, wie kommunalen Interessenverbänden, Einfluss nehmen zu können, müsse der AdR seine Informationen frühzeitig erhalten und gut vernetzt sein. Stahl wies auf das Weißbuch des AdR zu ,multi-level governance“ hin, das im Juni 2009 angenommen wurde. Ziel des AdR sei es, eine „Multi-Level-GovernanceCharta“ der Europäischen Union auszuarbeiten. Als Beispiele für eine funktionierende ,multilevel governance" nannte Stahl etwa die Monitoring-Plattform des AdR für die Lissabon-Strategie, das Netz für Subsidiaritätskontrolle und die Ostseestrategie der Europäischen Union.

Ausgewählte Politikfelder im EU-Mehrebenensystem

Die demografische Entwicklung der Europäischen Union und die daraus entstehenden 
Herausforderungen wurden von Ole Wintermann dargestellt. Die Bevölkerung werde bis 2060 in Asien auf über fünf Milliarden und in Afrika auf über zwei Milliarden Menschen steigen. Die anderen Weltregionen blieben in der Zahl ihrer Einwohner relativ stabil beziehungsweise wüchsen nur leicht. Angesichts dieser sich abzeichnenden demografischen Trends werde Europa in der Welt von 2060 relativ an Bedeutung verlieren. Entsprechend müsse die europäische Ebene an Bedeutung gewinnen, weil die einzelnen Mitgliedstaaten an Einfluss verlören. Innerhalb Europas werde Deutschland dann, was die Bevölkerungszahl angehe, von der Türkei, Großbritannien und Frankreich überholt werden. Die Türkei werde 2060 fast 100 Millionen Einwohner haben, Großbritannien fast 80 Millionen. Wie Wintermann betonte, sei Bevölkerungswachstum eher eine regionale als eine nationale Größe. Es gäbe in Europa und in Deutschland zahlreiche Studien zum demografischen Wandel und den Konsequenzen. Daraus folge bislang keine politische Aktivität. Die hohe Staatsverschuldung in Deutschland schränke die Handlungsspielräume für die Bewältigung der demografischen Probleme in den Sozialsystemen erheblich ein. Wenn man die Demografie als Faktor zur Berechnung des Wirtschaftswachstums einbeziehe, dann werde die deutsche Volkswirtschaft in den nächsten Jahrzehnten schrumpfen. Das hätte ebenfalls gravierende Auswirkungen für die mittel- und langfristige Finanzplanung in Deutschland.

Klimapolitik als ein Teil der Umweltpolitik kann nach dem Vortrag von Annegret Eppler als Beispiel für das Handeln in einem ,multilevel governance'-System dienen. Weitgehend unstrittig sei, dass der Klimawandel eine der globalen Herausforderungen der nächsten Jahrzehnte darstelle. An der Gestaltung der Klimapolitik seien alle politischen Ebenen, von der internationalen bis zur lokalen Ebene, beteiligt. Die Europäische Union sei auf der internationalen Ebene als ein maßgeblicher Akteur anerkannt und betreibe eigene klimapolitische Programme. Die kommunale Ebene werde ebenfalls in der Klimapolitik aktiv. Es gebe auch eine Vernetzung der Kommunen auf der europäischen Ebene, wie zum Beispiel das Klimabündnis der europäischen Städte zeige. Eppler zeigte auf, dass an der Klimapolitik auf allen Ebenen zahlreiche staatliche und nichtstaatliche Akteure beteiligt seien, beispielsweise Wissenschaftler, Umwelt- und Wirtschaftsverbände, Unternehmen und die Zivilbevölkerung. Die Bandbreite der Steuerungsmittel reiche von regulativen Instrumenten über ökonomische Anreize bis hin zu freiwilligen Selbstverpflichtungen und Mitwirkungsmöglichkeiten der Bevölkerung. Eppler regte an, das Beispiel des Zusammenwirkens verschiedener Akteure und Steuerungsmittel der Klimapolitik auf andere Politikfelder zu übertragen. Im Hinblick auf die Reflexionsgruppe merkte sie an, dass auch in der Stellungnahme zu Politikinhalten - wie es dem Mandat der Reflexionsgruppe entspreche - ein Bild von der Art des Regierens gezeichnet werden könne, durch das auf Strukturen und Prozesse verwiesen werde.

Ute Behning plädierte gleich zu Anfang ihres Vortrags für ein zugkräftiges Thema, um die Integration der Europäischen Union weiter voranzutreiben. Ein solches Zugpferd könne die Sozialpolitik sein. Eine europäische Sozialpolitik sei ihrer Ansicht nach notwendig, um die bestehende Wirtschafts- und Währungsunion zu ergänzen. Gemeinsame Standards im Bereich der Sozialpolitik seien notwendig, um eine innereuropäische Konkurrenz bei den Sozialstandards zu verhindern (,Sozialdumping '). Der Vertrag von Lissabon ermögliche es, sozialpolitische Maßnahmen auf der europäischen Ebene zu beschließen. Die genaue Kompetenzabgrenzung zwischen der europäischen Ebene und den anderen Akteuren des Mehrebenensystems sei noch unklar. In der Beschäftigungspolitik bleibe die Kompetenz der Nationalstaaten bestehen.

Über Kommunen und die politische Unterstützung der Europäischen Union durch die Bürger sprach Angelika Vetter. Politische Unterstützung als eine Grundvoraussetzung für 
das Funktionieren eines politischen Systems speise sich aus der Bewertung der Leistungen (,output'), der Bewertung der zugrundeliegenden Werte und der emotionalen Verbundenheit mit einem politischen System. Bürger verfügten heute über multiple Identitäten und fühlten sich gleichzeitig unterschiedlichen Ebenen des Mehrebenensystems verbunden. Die Verbundenheitsgefühle gegenüber der Nation und der Kommune lägen dabei deutlich höher als die gegenüber der Europäischen Union. Etwa fünfzig Prozent der Unionsbürger fühlten sich der Europäischen Union verbunden. Mit ihrer Nation fühlten sie sich zu mehr als neunzig Prozent verbunden. Vetter stellte die Frage: Wie könnten Kommunen die Verbundenheit der Bürger mit der Europäischen Union fördern? Die politischen und gesellschaftlichen Eliten sollten einen positiven Diskurs über die Europäische Union pflegen. Die Einflussmöglichkeiten der Kommunen auf europäischer Ebene sollten wahrgenommen und kommuniziert werden. Gleichzeitig sollten die Kommunen europäische Entscheidungen, die von den Bürgern positiv wahrgenommen würden, auch als europäische Entscheidungen kommunizieren. Bislang würden positive Entwicklungen aus Brüssel dem Bürger vielfach als lokale Erfolge verkauft. Negativ wahrgenommene Entscheidungen der Europäischen Union würden hingegen von den Kommunen auch als EU-Entscheidungen dargestellt. Damit entstehe beim Bürger eine falsche Wahrnehmung der Politik der Europäischen Union. Sie kam zu dem Schluss, dass Kommunen zu einer positiven Bewertung der Europäischen Union beitragen könnten. Allerdings dürfe nicht übersehen werden, dass die Entwicklung politischer Unterstützung ein langfristiger Prozess sei. Die Kommunen könnten hier eine verantwortungsvolle Rolle im politischen System der Europäischen Union übernehmen und dürften keinesfalls überschätzt werden.

In der abschließenden Diskussion kamen Gabriele Abels und Michèle Knodt sowie die Mehrheit der Teilnehmer zu dem Ergebnis, dass die Bedingungen für einen Erfolg der Reflexionsgruppe „Horizont 2020-2030“ schlecht seien. Ohne eine Überdehnung des Mandats der Reflexionsgruppe würden kaum Chancen für ein Gelingen bestehen. Abels verwies auf die Agenda-Setting-Funktion von Reflexionsgruppen und empfahl der Reflexionsgruppe, sich durch die Einführung neuer Begriffe und Konzepte auf diese Funktion zu konzentrieren. Ferner empfahl sie der Reflexionsgruppe, bereits jetzt eine Debatte über die künftigen Ergebnisse sowohl mit einer breiteren Öffentlichkeit als auch mit strategisch wichtigen Akteuren zu beginnen, damit der Bericht rezipiert und breit diskutiert würde. Bezogen auf die Herausforderungen für die kommunale Ebene betonte Knodt, dass die Reflexionsgruppe für die zukünftige Arbeit der Europäischen Union ein ,regionales und kommunales Mainstreaming" konzeptionalisieren sollte. In der Umsetzung würde dies bei allen Politiken bedeuten, eine ,differenzierte regionale und kommunale Folgenabschätzung "durchzuführen, um als Frühwarnsystem die Folgen des Gemeinschaftshandelns für die regionale und kommunale Ebene zu prognostizieren. Kommunen sollten weiterhin ihre Europafähigkeit ausbauen und ihre Potenziale der Bürgernähe für die Generierung von europäischer Identifikation besser nutzen.

Eckart Stratenschulte empfahl der Reflexionsgruppe sich über den Adressaten ihrer Arbeit klar zu sein: die europäische Öffentlichkeit. Die Reflexionsgruppe müsse sich zuallererst Gedanken über ein europäisches Leitbild machen: Wie solle die Europäische Union 2030 aussehen? Aus diesem Leitbild folge dann eine Analyse der Situation im Jahre 2030 und der Faktoren, die der Erreichung dieses Leitbildes entgegenstünden. Erst dann könne ein Lösungsvorschlag der Reflexionsgruppe für eine dem Leitbild entsprechende Europäische Union im Jahr 2030 erfolgen. 